Pacific Journal of Mathematics

SIZED SURFACES WITH HYPERELLIPTIC SECTIONS

aldo Biancofiore, Maria Lucia mania and Antonio banter 


\section{POLARIZED SURFACES WITH HYPERELLIPTIC SECTIONS}

\section{Aldo Biancofiore, Maria lucia Fania, ANd Antonio Lanteri}

Using an argument by Castelnuovo we classify surfaces $X$ with an ample and spanned line bundle $L$ whose hyperelliptic locus is large enough.

Introduction. The classification of projective surfaces with hyperelliptic hyperplane sections has recently been accomplished by Sommese and Van de Ven [SVdV] and by Serrano [Se]. Letting $X$ be a complex projective non-singular algebraic surface and $H$ be the hyperplane bundle, the pairs $(X, H)$ as above with sectional genus $g=g(H) \geq 2$ are:

(i) scrolls over a hyperelliptic curve,

(ii) rational conic bundles,

(iii) a special geometric conic bundle over an elliptic curve,

(iv) a Del Pezzo surface of degree 2 with $H=K_{X}^{-2}$,

(v) a Del Pezzo surface of degree 1 with $H=K_{X}^{-3}$,

(vi) a single blow-up of a pair as in (iv).

In the first three cases all smooth elements of $|H|$ are hyperelliptic. This is obvious for (i) and (ii), while it is a recent discovery of Serrano for (iii). In the remaining cases the hyperelliptic locus of $|H|$ is a linear space of small codimension.

In this paper we carry out the classification of polarized surfaces whose hyperelliptic locus is large enough. More precisely, let $L$ be an ample and spanned line bundle on a surface $X$ and let $\mathscr{H}$ be the closure of the set of smooth hyperelliptic elements of $|L|$. We classify the pairs $(X, L)$ as above under the assumption that $\operatorname{dim} \mathscr{H} \geq 2$. We need this technical assumption to rephrase in our context a classical argument by Castelnuovo consisting in putting together the $g_{2}^{1}$ 's of all elements of $\mathscr{H}_{0}$, an irreducible 2 -dimensional component of $\mathscr{H}$, to express $X$ itself as a double cover.

This assumption is largely satisfied when $L$ is very ample.

Our main result can be stated as follows.

Assume that $\operatorname{dim} \mathscr{H} \geq 2$. Then either $(\mathrm{I})(X, L)$ is a scroll over 
a hyperelliptic curve, or (II) $K_{X} \otimes L$ is spanned, or possibly (III) $X$ is some special ruled surface with $c_{1}(L)^{2} \leq 4$. In case (II) either (a) $(X, L)$ is a conic bundle, or (b) up to a reduction, there exists a morphism $\pi: X \rightarrow Y$ of degree 2 onto a normal surface $Y$, which is one of the following: $\left(b_{1}\right) \mathbf{P}^{2}$ or the Veronese surface, $\left(b_{2}\right)$ a rational normal scroll, $\left(b_{3}\right)$ the quadric cone, and $L=\pi^{*} \mathscr{O}_{Y}(1)$.

It should be noted that the 2-dimensional pairs considered by Fujita in [F2] fall into our classification.

Note that (ii) and (iii) fit into (a), (iv) into $\left(b_{1}\right),(v)$ into $\left(b_{2}\right)$, while (vi) shows the necessity of the reduction in (b).

The paper is organized as follows.

In $\S 1$ we prove the structure theorem when $c_{1}(L)^{2} \geq 5$.

In $\S 2$ we complete our study when $c_{1}(L)^{2} \leq 4$ through a case by case analysis using the morphism associated to $|L|$.

In $\S 3$ we make a more detailed analysis of conic bundles under the assumption $c_{1}(L)^{2} \geq 5$. If $\mathscr{H} \neq \varnothing$, then the adjunction mapping maps $X$ onto $\mathbf{P}^{1}$ and factors through the ruling $\alpha: X \rightarrow B$ of $X$ and a finite morphism $\beta: B \rightarrow \mathbf{P}^{1}$. By adapting an argument by Sommese [So1], we show that either $\beta$ is an embedding or $c_{1}(L)^{2} \leq 8, \beta$ has degree 2 and $B$ is either elliptic or hyperelliptic.

In $\S 4$ we provide an application to vector bundles. Let $E$ be an ample and spanned rank-2 holomorphic vector bundle with $c_{2}(E)=2$, on a smooth surface $X$. Then we show that either $(X, E)$ is one of the following pairs: $\left(\mathbf{P}^{2}, \mathscr{O}_{\mathbf{P}^{2}}(1) \oplus \mathscr{O}_{\mathbf{P}^{2}}(2)\right),\left(\mathbf{Q}, \mathscr{O}_{\mathbf{Q}}(1)^{\oplus 2}\right)$, where $\mathbf{Q} \subset \mathbf{P}^{3}$ is a quadric surface, or $\pi: X \rightarrow \mathbf{P}^{2}$ is a double cover and $E=$ $\left(\pi^{*} \mathscr{O}_{\mathbf{P}^{2}}(1)\right)^{\oplus 2}$, or, possibly, $(X, \operatorname{det} E)$ is a geometric conic bundle over an elliptic or hyperelliptic curve as in $\S 3$. Unfortunately we do not know whether the latter possibility does really occur.

The above result generalizes what the third author proved in [L1] and [L2]. A similar result has been obtained by Ballico [Ba] in a different way.

We are indebted to the referee for his useful observations.

\section{Preliminaries.}

(0.0) Throughout this paper $X$ will denote a smooth complex connected projective surface and $L \in \operatorname{Pic}(X)$ an ample and spanned line bundle (i.e. spanned by its global sections). We let $d=c_{1}(L)^{2}=L \cdot L$ and

$$
g=1+\frac{1}{2}\left(L \cdot\left(L \otimes K_{X}\right)\right)
$$

where $K_{X}$ stands for the canonical bundle. 
We shall always assume that $g \geq 2$.

A pair $(X, L)$ as above is said to be a scroll if $X$ is a $\mathbf{P}^{1}$-bundle over a smooth curve and $\left.L\right|_{f}=\mathscr{O}_{\mathbf{P}^{\prime}}(1)$ for every fibre $f$ of $X$. A pair $(X, L)$, which is not a scroll, is a conic bundle if $X$ is a ruled surface and $\left.L\right|_{F}=\mathscr{O}_{\mathbf{P}^{\prime}}(2)$ for the general fibre $F$ of the ruling.

If $X$ is a $\mathbf{P}^{1}$-bundle we denote by $\zeta$ a fundamental section, and by $f$ a fibre; $e=-\zeta \cdot \zeta$ will denote the invariant of $X$. We let $\mathbf{F}_{e}=\mathbf{P}\left(\mathscr{O}_{\mathbf{P}^{1}} \oplus \mathscr{O}_{\mathbf{P}^{1}}(-e)\right)$.

As known (e.g. see [So1]) if $L$ is very ample then $K_{X} \otimes L$ is spanned unless $(X, L)$ is a scroll. For ample and spanned line bundles we have the following

(0.1) Proposition. Let $d \geq 5$; then $K_{X} \otimes L$ is spanned unless $(X, L)$ is a scroll.

Proof. By Reider's theorem [R] if $K_{X} \otimes L$ is not spanned, then $X$ contains an effective divisor $E$ satisfying either

$$
L \cdot E=0 \text { with } E^{2}=-1 \text { or } L \cdot E=1 \text { with } E^{2}=0 .
$$

The former case cannot occur since $L$ is ample. In the second case $E$ is irreducible.

Consider the map $f: X \rightarrow f(X) \subset \mathbf{P}$ associated to $|L|$. Since $L$ is ample and spanned, $f$ is a morphism and $\operatorname{dim} f(X)=2$. Then the equality

$$
1=L \cdot E=\left.\operatorname{deg} f\right|_{E} \cdot \operatorname{deg} f(E)
$$

implies that $f(E)$ is a line and $\left.f\right|_{E}$ an isomorphism. Therefore $E \approx$ $\mathbf{P}^{1}$; hence $X$ is ruled as $E^{2}=0$ and $(X, L)$ is a scroll.

(0.2) REMARK. The assumption $d \geq 5$ is crucial in $(0.1)$ as the following example shows. $X=\mathbf{P}^{1}$-bundle of invariant $e=-1$ over an elliptic curve, $L=[2 \zeta] . L$ is ample and spanned, $d=4$; however $h^{0}\left(K_{X} \otimes L\right)=1$.

Let $d \geq 5$; if $(X, L)$ is not a scroll we can consider the adjunction mapping $\Phi$, i.e. the map associated to $K_{X} \otimes L$, which is a morphism by $(0.1)$.

(0.3) Proposition ([LP1]). Assume that $K_{X} \otimes L$ is spanned and that $g \geq 2$. The following facts are equivalent:

- $\left(K_{X} \otimes L\right) \cdot\left(K_{X} \otimes L\right)=0$, 
- $(X, L)$ is a conic bundle.

$-\operatorname{dim} \Phi(X)=1$.

We need to recall the concept of reduction.

(0.4) A curve $E \approx \mathbf{P}^{1}$ contained in $X$ is said to be a $(-1)$ line relative to $L$ if $E \cdot E=-1$ and $[E] \cdot L=1$. As it is known, $X$ contains a finite number of $(-1)$ lines relative to $L$ and if $(X, L)$ is not a conic bundle, all such curves are disjoint. Assume that $(X, L)$ is not a conic bundle and let $\eta: X \rightarrow X^{\prime}$ be the birational morphism contracting all the $(-1)$ lines relative to $L$ of $X$. Then $X^{\prime}$ is a smooth surface and $L^{\prime}=\eta_{*} L$ is an ample line bundle. The pair $\left(X^{\prime}, L^{\prime}\right)$ is usually referred to as the reduction of $(X, L)$. Note that

$$
K_{X} \otimes L=\eta^{*}\left(K_{X^{\prime}} \otimes L^{\prime}\right) .
$$

So, if $K_{X} \otimes L$ is spanned and $\operatorname{dim} \Phi(X)=2$, the adjunction map $\Phi$ factors through the reduction morphism $\eta$.

1. Structure theorem. Let $(X, L)$ be as in $(0.1)$. We let

$$
\mathscr{H}=\overline{\{C \in|L|, C \text { smooth hyperelliptic }\}}
$$

and we will refer to $\mathscr{H}$ as the hyperelliptic locus of $(X, L)$. We will assume that

$$
\operatorname{dim} \mathscr{H} \geq 2 .
$$

If $(X, L)$ is a scroll, then $K_{X} \otimes L$ is not spanned. If $\mathscr{H} \neq \varnothing$, then $X$ admits a hyperelliptic curve as a section; this implies that the base curve of $X$ is hyperelliptic and then $\mathscr{H}=|L|$. So, an obvious class of pairs satisfying (1.0) consists of

(1.1) scrolls over a hyperelliptic curve.

Let $\mathscr{H}_{0}$ be an irreducible component of $\mathscr{H}$ of $\operatorname{dim} \mathscr{H}_{0} \geq 2$. Let $p \in X$ and let $|L-p|$ be the sublinear system of elements of $|L|$ passing through $p$. We let

$$
\mathscr{H}_{0}(p)=\mathscr{H}_{0} \cap|L-p| .
$$

Note that $\mathscr{H}_{0}(p)$ is connected since $|L-p|$ is a hyperplane of $|L|$. Let $\Gamma(p) \subset X \times \mathscr{H}_{0}(p)$ be the closure of the set

$$
\left\{\left(p^{\prime}, C\right) \in X \times \mathscr{H}_{0}(p): i_{c}(p)=p^{\prime}\right\},
$$

where $i_{c}$ stands for the hyperelliptic involution of $C . \Gamma(p)$ is connected and its image $\gamma(p)$ under the projection onto the first factor is also so. 
(1.2) If $\operatorname{dim} \gamma(p)=0$, then $\gamma(p)$ consists of a single point.

Now assume that $K_{X} \otimes L$ is spanned. Since

$$
\gamma(p) \subset \Phi^{-1}(\Phi(p))
$$

if $\operatorname{dim} \gamma(p)>0$ we see that either

(1.2.1) $(X, L)$ is a conic bundle and $\gamma(p)$ is contained in a fibre of it, or

(1.2.2) $\operatorname{dim} \Phi(X)=2$ and $\gamma(p)$ is a $(-1)$ line relative to $L$.

(1.2.1) follows from (0.3) and (1.2.2) from (0.4).

(1.3) Theorem. Assume that $K_{X} \otimes L$ is spanned and that (1.0) holds. Then either

(i) $(X, L)$ is a conic bundle, or

(ii) up to a reduction there exists a morphism of degree two $\pi: X \rightarrow$ $Y$ on a normal, possibly singular surface $Y, L=\pi^{*} M$ and the pair $(Y, M)$ is one of the following:

(1.3.1) $\left(\mathbf{P}^{2}, \mathscr{O}_{\mathbf{P}^{2}}(e)\right), e=1,2$,

(1.3.2) $\left(\mathbf{F}_{e},\left.M\right|_{f}=\mathscr{O}_{\mathbf{P}^{1}}(1)\right)$,

(1.3.3) $\left(\widetilde{\mathbf{F}}_{2}, \nu^{*} M=[\zeta+2 f]\right)$ (here $\widetilde{\mathbf{F}}_{e}$ stands for $\mathbf{F}_{e}$ with the fundamental section $\zeta$ collapsed and $\nu: \mathbf{F}_{e} \rightarrow \widetilde{\mathbf{F}}_{e}$ for the desingularization). Moreover the adjunction mapping factors through $\pi$.

Proof. If $\operatorname{dim} \gamma(p)=0$ for every $p \in X$, then due to (1.2) there is an involution $i$ on $X$. Let $\pi: X \rightarrow Y=X /\langle i\rangle$ be the obvious morphism and note that $Y$ is a normal surface and $L=\pi^{*} M$ for some $M=\mathscr{O}_{Y}(D), D$ being a Weil divisor. Moreover $\pi(C)=C /\left\langle i_{c}\right\rangle$ is a smooth $\mathbf{P}^{1}$ contained in $Y$ as an ample divisor. Hence the pair $(Y, M)$ is either as in (1.3.1), (1.3.2) or $\left(\widetilde{\mathbf{F}}_{e}, \nu^{*} M=[\zeta+e f]\right), e \geq 1$, (e.g. see [Sa], [So2]). Note that $e \geq 2$, otherwise we would be in case (1.3.1). Now consider the commutative diagram

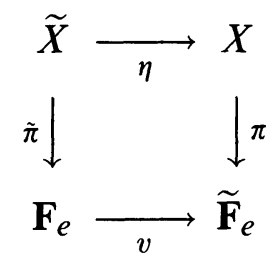

where $\tilde{X}=X \times_{\widetilde{\mathbf{F}}_{e}} \mathbf{F}_{e}$ and $\tilde{\pi}$ is the double cover induced by $\pi$. 
Since $X$ is smooth, $\eta$ collapses $\tilde{\pi}^{-1}(\zeta)$ to a finite set. $\widetilde{\mathbf{F}}_{e}$.

Claim. The branch locus of $\pi$ contains $x$, the vertex of the cone

If this were not true, i.e. $\pi^{-1}(x)=x^{\prime} \cup x^{\prime \prime}, x^{\prime} \neq x^{\prime \prime}$, then $(\tilde{\pi})^{*} \zeta=$ $E^{\prime}+E^{\prime \prime}$, where $\eta\left(E^{\prime}\right)=x^{\prime}, \eta\left(E^{\prime \prime}\right)=x^{\prime \prime}$ and $E^{\prime} \cdot E^{\prime \prime}=0$. Thus

$$
-2=\left(E^{\prime}+E^{\prime \prime}\right)^{2}=2 \zeta^{2}=-2 e
$$

which gives $e=1$, contradiction.

From the claim it follows that $\tilde{\pi}$ is ramified along the $(-1)$ curve $E=\eta^{-1}\left(\pi^{-1}(x)\right)$; hence $(\tilde{\pi})^{*} \zeta=2 E$ which gives $e=2$, i.e. $(Y, M)$ is as in (1.3.3).

Now assume that $\operatorname{dim} \gamma(p)=0$ for the general $p \in X$. Then in view of (1.2.2) there is a reduction $\left(X^{\prime}, L^{\prime}\right)$ of $(X, L)$ such that $\operatorname{dim} \gamma(p)=0$ for every $p \in X^{\prime}$ and then the above argument gives (ii).

Finally if $\operatorname{dim} \gamma(p)>0$ for the general $p \in X$, then case (i) occurs in view of (1.2.1).

The last assertion follows from the fact that $p$ and $\gamma(p)$ are in the same fibre of $\Phi$.

(1.4) Remark. Assume that $L$ is very ample and $\mathscr{H} \neq \varnothing$. Then according to [SVdV] (see also [Se]), apart from scrolls and conic bundles, $(X, L)$ has to be one of the following pairs:

(a) $X$ is a Del Pezzo surface of degree 2 and $L=K_{X}^{-2}$,

(b) $X$ is a Del Pezzo surface of degree 1 and $L=K_{X}^{-3}$,

(c) $(X, L)$ has a simple reduction as in (a).

In case (a), $K_{X} \otimes L=K_{X}^{-1}$ is spanned and the adjunction mapping exhibits $X$ as a double cover $\pi: X \rightarrow \mathbf{P}^{2}$; then $L=\pi^{*} \mathscr{O}_{\mathbf{P}^{2}}(2)$ and $\mathscr{H}=\pi^{*}\left|\mathscr{O}_{\mathbf{P}^{2}}(2)\right| ;$ this fits into case (1.3.1). Note also that $\left(X, K_{K} \otimes L\right)$ fits into case (1.3.1).

In case (b) $X$ is the blow-up of $\mathbf{P}^{2}$ at 8 points $p_{1}, \ldots, p_{8}$ in general position and $|L|$ corresponds to the linear system $\mid \mathscr{O}_{\mathbf{P}^{2}}(9)-$ $3 p_{1}-\cdots-3 p_{8} \mid$ of conics having triple points at $p_{1}, \ldots, p_{8}$. Let $p_{9}$ be the further base point of the pencil of cubics through $p_{1}, \ldots, p_{8}$. Then $\mathscr{H}$ is the hyperplane of $|L|$ corresponding to the linear system $\mathscr{S}=\left|\mathscr{O}_{\mathbf{P}^{2}}(9)-3 p_{1}-\cdots-3 p_{8}-p_{9}\right|$. Actually the pencil cuts out a $g_{2}^{1}$ on every smooth curve $C \in \mathscr{S}$ out of $p_{9}$. Then it is easy to see that $\mathscr{H}$ defines a map $X \rightarrow \mathbf{P}^{5}$ of degree 2 onto the quartic rational scroll of $\mathbf{P}^{5}$, isomorphic to $\mathbf{F}_{2}$. So $(X, L)$ is as in (1.3.2). Note that $K_{X} \otimes L=K_{X}^{-2}$ is ample and spanned and the adjunction mapping 
expresses $X$ as a double cover of the quadric cone $\widetilde{\mathbf{F}}_{2}$, branched at the vertex and along the transverse intersection with a cubic surface. In other words $\left(X, K_{X} \otimes L\right)$ is as in (1.3.3).

Finally case (c) shows the necessity of the reduction in (1.3).

2. Small degrees. In this section, regardless of whether $K_{X} \otimes L$ is spanned or not we look at the cases $d \leq 4$, under the assumption (1.0).

Let $f$ be the morphism associated to $|L|$ and let $Z=f(X)$. Then we have

$$
4 \geq d=\operatorname{deg} f \cdot \operatorname{deg} Z \geq \operatorname{deg} f \cdot\left(h^{0}(L)-2\right) .
$$

Note also that $h^{0}(L) \geq 3$, since $L$ is ample and spanned. Moreover if $\operatorname{deg} f=1, Z$ cannot have isolated singular points due to the ampleness of $L$.

By (2.0.1) and the well known classification of polarized surfaces of $\Delta$-genus $\leq 1$ (e.g. see [F1]), recalling that $g \geq 2$ we get only the following possibilities for $(X, L)$ :

(1) $d=2, f: X \rightarrow \mathbf{P}^{2}$ a double covering branched along a smooth curve of degree $2 n$ with $n \geq 3$,

(2) $d=3, f: X \rightarrow \mathbf{P}^{2}$ a triple cover,

(3) $d=4, f: X \rightarrow \mathbf{P}^{3}$ generically of degree 1 ,

(4) $d=4, f: X \rightarrow \mathbf{Q} \subset \mathbf{P}^{3}$ a double cover of a quadric,

(5) $d=4, f: X \rightarrow \mathbf{P}^{2}$ a 4-tuple cover.

Case (1) is included in (1.3.1) while case (4) is included in (1.3.2) or (1.3.3) according to whether $\mathbf{Q}$ is a smooth quadric or a cone.

To deal with cases (2) and (3) we need to recall the following result:

(2.1) THEOREM ([LP2, Th. 3.1]). Let $X$ be a surface polarized by an ample and spanned line bundle $L$, with $g(L)=2$. Then $(X, L)$ is one of the following pairs:

(2.1.1) a scroll over a smooth curve of genus two;

(2.1.2) $X$ is a $\mathbf{P}^{1}$-bundle over an elliptic curve, with invariant $e=-1$ and $L \sim_{n}[2 \zeta]$;

(2.1.3) $X$ is an $\mathbf{F}_{e}(e \leq 2)$ blown-up at a finite set $P$ consisting of $s \leq 9$ points on discrete fibres and $L=\sigma^{*} L_{0} \otimes[E]^{-1}$, where $\sigma: X \rightarrow \mathbf{F}_{e}$ is the blow-up, $E=\sigma^{-1}(P)$ and $L_{0}=[2 \zeta+(e+3) f]$;

(2.1.4) $\pi: X \rightarrow \mathbf{P}^{2}$ is the $K 3$ double cover branched along a smooth sextic and $L=\pi^{*} \mathscr{O}_{\mathbf{P}^{2}}(1)$; 
(2.1.5) $\pi: X \rightarrow \mathbf{Q} \subset \mathbf{P}^{3}$ is a double cover of a quadric cone branched at $a$ vertex and along the transverse intersection of $\mathbf{Q}$ with a cubic surface and $L=\pi^{*} \mathscr{O}_{\mathbf{Q}}(1)$.

Since [LP2] is not yet published, we give a sketchy proof for the convenience of the reader. Let $C \in|L|$ be a general element. Since $C$ has genus 2 , the 2-canonical bundle of $C$ is not very ample. Hence, by adjunction, $\left(K_{X} \otimes L\right)^{2}$ cannot be birationally very ample. As $\left(K_{X} \otimes L\right)^{2}=K_{X} \otimes M$, where $M=K_{X} \otimes L^{2}$, we see that either

(a) $K_{X} \otimes L^{2}$ is not very ample, or

(b) $M$ is very ample, but $K_{X} \otimes M$ is not birationally very ample. By using Reider's theorem [R], case (a) immediately leads to (2.1.1) and (2.1.4). In case (b) we have to use [SVdV]. Then cases (2.1.2), (2.1.3), (2.1.5) come by checking the list of the exceptions in Theorems $(0.1)$ and $(2.1)$ of $[\mathbf{S V d V}]$ for $(X, M)$. Some care is needed when $(X, L)$ is a conic bundle.

Now come back to our cases.

In case (3), $Z$ is a quartic surface and $f$ induces a birational map between the general $C \in|L|$ and the hyperplane section of $Z$. Since the only hyperelliptic plane curves of degree 4 and $g \geq 2$ are quartics with a single double point we conclude that $Z$ can only be a quartic surface with a double line. Then $(X, L)$ is as in (2.1.3) with $s=8$.

In case (2) let $C \in \mathscr{H}$ be a general element and consider the morphism

$$
\varphi: C \rightarrow \mathbf{P}^{1} \times \mathbf{P}^{1}
$$

given by the $g_{2}^{1}$ of $C$ and $g_{3}^{1}$ induced by $\left.f\right|_{C}$. The image $C^{\prime}=\varphi(C)$ has bidegree $(2,3)$. Note that $\varphi$ is generically one to one since 2 and 3 are relatively prime. The arithmetic genus of $C^{\prime}$ is $p_{a}(C)=2$; hence $g=2$ as $2 \leq g \leq p_{a}\left(C^{\prime}\right)$. Then $(X, L)$ can only be as in (2.1.1) or (2.1.3) with $s=9$.

In case (2.1.1) a few computations using the ampleness and the spannedness assumption provide only the following possibility: $X$ has invariant $e=-1, L \sim_{n}[\zeta+2 f]$ and the restriction of $L$ to every fundamental section is the hyperelliptic bundle.

In case (5) we have $\mathscr{H}=|L|$ due to our assumption (1.0). Let $C \in$ $\mathscr{H}$ be a general element and consider the morphism $\varphi: C \rightarrow \mathbf{P}^{1} \times \mathbf{P}^{1}$ given by the $g_{2}^{1}$ of $C$ and $\left.f\right|_{C}$ and let $C^{\prime}=\varphi(C)$.

There are two possibilities, either

(i) $\varphi$ is generically one to one and $C^{\prime}$ has bidegree $(2,4)$ or

(ii) $\varphi$ is generically 2 to 1 . 
In case (ii) $\left.f\right|_{C}$ factors through the $g_{2}^{1}$ of $C$. If this happens for the general $C \in \mathscr{H}$, then a global involution on $X$ is defined. Since $\mathscr{H}=|L|$ by (1.0) it follows that $f$ itself factors through a morphism $\pi: X \rightarrow Y$ of degree 2 onto a normal surface $Y$ and $L=\pi^{*} M$, where $M=\mathscr{O}_{Y}(D)$. Moreover $(Y, M)$ is as in (1.3.3) or (1.3.2) with $e=0$. Now assume that there is a smooth $C \in \mathscr{H}$ as in case (i). Since $C^{\prime}$ has bidegree $(2,4)$ we have $p_{a}\left(C^{\prime}\right)=3$ and so $g \leq 3$.

If $g=2$, then $(X, L)$ is as in (2.1.1) or (2.1.2).

In case (2.1.1) an immediate computation using the ampleness and the spannedness assumptions shows that $X$ can only have invariant $e=-2$ or $0, L \sim_{n}[\zeta+(2+e / 2) f]$ and if $e=0$ the restriction of $L$ to every fundamental section is the hyperelliptic bundle.

If $g=3$ then $K_{X} \cdot L=0$ by the genus formula. So either $X$ is ruled or it is a minimal surface of Kodaira dimension 0 . In the latter case, since $h^{0}(L)=3$, from the Riemann-Roch theorem we get $\chi\left(\mathscr{O}_{X}\right)=1$; hence $X$ is an Enriques surface. This case however cannot occur (see [V, Prop. 1.1]).

Assume that $X$ is ruled. As a first thing $(X, L)$ can be a scroll over a hyperelliptic curve of genus three. Then an easy computation shows that $X$ has invariant $e=-2$ or 0 and $L \sim_{n}[\zeta+(2+e / 2) f]$ and if $e=0$ the restriction of $L$ to every fundamental section is the hyperelliptic bundle. If $(X, L)$ is not a scroll, then

$$
K_{X} \cdot K_{X}-4=\left(K_{X} \otimes L\right) \cdot\left(K_{X} \otimes L\right) \geq 0,
$$

with equality iff $(X, L)$ is a conic bundle [LP1]. In this case one can see that $X$ is obtained by blowing-up $\mathbf{F}_{e}(e \leq 3)$ at 12 points $p_{1}, \ldots, p_{12}$ on distinct fibres and

$$
L=\eta^{*}[2 \zeta+(e+4) f] \otimes\left[E_{1}+\cdots+E_{12}\right]^{-1}
$$

where $\eta: X \rightarrow \mathbf{F}_{e}$ is the blowing-up and $E_{i}=\eta^{-1}\left(p_{i}\right)$. If $(X, L)$ is neither a scroll nor a conic bundle, then $K_{X} \cdot K_{X} \geq-3$. On the other hand, since $X$ is ruled, by the Hodge index theorem we get $K_{X} \cdot K_{X} \leq-1$, so

$$
-3 \leq K_{X} \cdot K_{X} \leq-1 .
$$

Let $\left(X^{\prime}, L^{\prime}\right)$ be the reduction of $(X, L)$; then $K_{X^{\prime}} \otimes L^{\prime}$ is ample [LP1]. Note that $g\left(K_{X} \otimes L\right)=g\left(K_{X^{\prime}} \otimes L^{\prime}\right)=3+K_{X} \cdot K_{X}+K_{X}$. $L=3+K_{X} \cdot K_{X}$; so if $K_{X} \cdot K_{X}=-3$ or -2 , then $(X, L)$ can be recovered from the classification of polarized surfaces of sectional genus $\leq 1$, while, if $K_{X} \cdot K_{X}=-1$, then $g\left(K_{X} \otimes L\right)=2$, but $\left(K_{X}^{2} \otimes L\right)$. $\left(K_{X}^{2} \otimes L\right)=0$, so that $\left(X, K_{X} \otimes L\right)$ is a conic bundle. In all the cases, 
the irregularity of $X$ is $q=0$ or 1 and the structure of $(X, L)$ can be determined explicitly. For more details see [LL, sec. 2]. In this latter case it does not seem easy to decide whether $(X, L)$ does really satisfy the basic assumption (1.0).

3. More on conic bundles. In this section we study case (i) of (1.3). In view of $\S 2$ we can assume $d \geq 5$.

(3.1) LemMA. Let $(X, L)$ be a conic bundle. If $\mathscr{H} \neq \varnothing$, then $\Phi(X) \approx \mathbf{P}^{1}$.

Proof. Let $C \in|L|$ be a hyperelliptic curve. Then $\Phi$ maps $C$ onto a $\mathbf{P}^{1}$ since $\Phi(C)=C /\left\langle i_{c}\right\rangle$, where $i_{c}$ is the hyperelliptic involution of $C$. It thus follows from $(0.3)$ that $\Phi(X) \approx \mathbf{P}^{1}$.

Let $(X, L)$ be a conic bundle. We recall that the Stein factorization of the adjunction mapping is

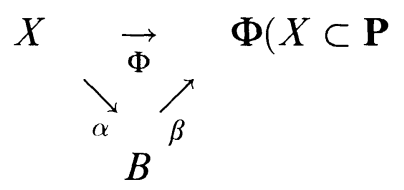

where $\alpha: X \rightarrow B$ is the ruling projection. So (3.1) implies that if $\beta$ is an embedding and $\mathscr{H} \neq \varnothing$, then $X$ is rational.

The main point of this section is the following proposition, extending a result proved by Sommese for very ample line bundles [So1, (2.1.1)].

(3.2) Proposition. Let $(X, L)$ be a nonrational conic bundle with $d \geq 5$ and $\mathscr{H} \neq \varnothing$. If $\beta$ is not an embedding then $d \leq 8, g=2 q+1$, $B$ is elliptic or hyperelliptic and $\beta: B \rightarrow \mathbf{P}^{1}$ has degree 2 .

Proof. Since $K_{X} \otimes L=\Phi^{*} \mathscr{O}_{\Phi(X)}(1)$, there is a line bundle $\mathscr{N} \in$ $\operatorname{Pic}(B)$ such that $K_{X} \otimes L=\alpha^{*} \mathscr{N}$. Moreover, $\operatorname{deg} \mathscr{N} \leq 2 q$ as $\beta$ is not an embedding. On the other hand, for a smooth $C \in|L|$ we have

$$
2 g-2=\left.\operatorname{deg}\left(K_{X} \otimes L\right)\right|_{C}=\left.\operatorname{deg} \alpha\right|_{C} \cdot \operatorname{deg} \mathscr{N} .
$$

This combined with the previous inequality gives

$$
g \leq 2 q+1 .
$$

Furthermore, since $(X, L)$ is a nonrational conic bundle, from $(0.3)$ it follows that

$$
\begin{aligned}
0 & =\left(K_{X} \otimes L\right) \cdot\left(K_{X} \otimes L\right) \\
& =K_{X} \cdot K_{X}+4 g-4-d \leq 8(1-q)+4 g-4-d
\end{aligned}
$$


this implies

$$
g \geq 2 q-1+d / 4
$$

So (3.2.1), (3.2.3) imply that $d \leq 8$.

Since $d \geq 5,(3.2 .1),(3.2 .3)$ also give $g=2 q+1$. This last fact implies that

$$
h^{0}(\mathscr{N})=h^{0}\left(K_{X} \otimes L\right)=q+1
$$

and

$$
\operatorname{deg} \mathscr{N}=\frac{1}{2}(2 g-2)=2 q .
$$

So, looking at the morphism $\beta$, we get

$$
2 q=\operatorname{deg} \mathscr{N}=\operatorname{deg} \beta \cdot \operatorname{deg} \Phi(X) \geq q \operatorname{deg} \beta .
$$

Hence $\operatorname{deg} \beta \leq 2$. Since $\beta$ is not an embedding and $\Phi(X) \approx \mathbf{P}^{1}$ by (3.1), it follows that $\operatorname{deg} \beta=2$ and $B$ is elliptic or hyperelliptic.

(3.3) REMARKs. (1) It follows from (3.2) that all pairs $(X, L)$ occurring in (i) or (1.3) for $d \geq 9$ are rational conic bundles.

(2) Putting $g=2 q+1$ in (3.2.2) we get $K_{X} \cdot K_{X}=8(1-q)-(8-d)$. Hence $X$ is a $\mathbf{P}^{1}$-bundle $X^{\prime}$ blown-up at $s=8-d$ points on distinct fibres. Then $\left(X^{\prime}, L^{\prime}=\eta_{*} L\right)$ is a geometric conic bundle and $d^{\prime}=$ $c_{1}\left(L^{\prime}\right)^{2}=d+s=8$. Since $L^{\prime} \sim_{n}[2 \zeta+b f]$ for some integer $b$, the condition $d^{\prime}=8$ implies $b=e+2$ and the ampleness of $L^{\prime}$ implies $e \leq 1$.

(3) Let $X=X^{\prime}$, i.e. $d=8$. Then $L \sim_{n}[2 \zeta+(e+2) f]$. Since $\zeta$ is a hyperelliptic curve, the spannedness of $L$ implies that $L \cdot[\zeta]=$ $2-e \geq 2$, i.e. $e \leq 0$, with equality iff $\left.L\right|_{\zeta}$ is the hyperelliptic line bundle of $\zeta$. In particular, let $B$ be a hyperelliptic curve of any genus $q \geq 2$ (an elliptic curve respectively) and let $\mathscr{L}$ be its hyperelliptic line bundle (any line bundle of degree 2 respectively). Let $p$ and $q$ be the projections of $X=B \times \mathbf{P}^{1}$ onto the factors and consider the line bundle $L=p^{*} \mathscr{L} \otimes q^{*} \mathscr{O}_{\mathbf{P}^{1}}(2)$. Then $L$ is ample and spanned and $q$ exhibits any smooth element of $|L|$ as a double cover of $\mathbf{P}^{1}$. Hence $\mathscr{H}=|L|$. This example shows that all values of $q$ are allowable in (3.2). This is the main difference with the corresponding situation in case of very ample line bundles (e.g. see [So1, (2.1.1)] and [Se, (3.3)]).

4. An application to vector bundles. The aim of this section is to classify pairs $(X, E)$ where

(4.0) $X$ is a smooth surface and $E$ is an ample and spanned rank-2 holomorphic vector bundle such that

$$
c_{2}(E)=2 \text {. }
$$


Note that the line bundle $L=\operatorname{det} E$ is ample and spanned. So this generalizes the situation considered in $[\mathbf{L 2}, \S 2]$, where $L$ is assumed to be very ample.

(4.1) Lemma. Let $(X, E)$ be as in (4.0). Then $X$ cannot contain lines relative to $L$.

Proof. Let $\ell \subset X$ be such a line. Then

$$
\left.E\right|_{\ell}=\mathscr{O}_{\mathbf{P}^{1}}(a) \oplus \mathscr{O}_{\mathbf{P}^{1}}(b),
$$

where $a$ and $b$ are positive integers, by ampleness. Then

$$
1=L \cdot[\ell]=c_{1}\left(\left.L\right|_{\ell}\right)=a+b \geq 2,
$$

a contradiction.

In order to classify our pairs $(X, E)$ we will proceed according to the values of $g=g(L)$. First of all we deal with the case $g \leq 1$.

(4.2) Proposition. Let $(X, E)$ be as in (4.0) and $g \leq 1$. Then

$$
(X, E)=\left\{\begin{array}{l}
\left(\mathbf{P}^{2}, \mathscr{O}_{\mathbf{P}^{2}}(1) \oplus \mathscr{O}_{\mathbf{P}^{2}}(2)\right), \\
\left(\mathbf{F}_{0}, \mathscr{O}_{\mathbf{F}_{0}}(1) \oplus \mathscr{O}_{\mathbf{F}_{0}}(1)\right) .
\end{array}\right.
$$

Proof. In view of (4.1) and of the known classification of polarized surfaces with sectional genus $\leq 1$ (e.g. [LP1]), $(X, L)$ can only be one of the following pairs: $\left(\mathbf{F}_{0}, \mathscr{O}_{\mathbf{F}_{0}}(2)\right),\left(\mathbf{P}^{2}, \mathscr{O}_{\mathbf{P}^{2}}(2)\right),\left(\mathbf{P}^{2}, \mathscr{O}_{\mathbf{P}^{2}}(3)\right)$. Then the proof is the same as that of (2.3) in [L2].

Now we consider the case $g \geq 2$. Note that the assumption $c_{2}(E)=$ 2 is equivalent to saying that the general section $s \in \Gamma(E)$ vanishes exactly at two points of $X$. Let $s_{1}, s_{2}$ be two general elements of $\Gamma(E)$ and let $\ell \subset|E|=\mathbf{P}(\Gamma(E))$ be the line spanned by their classes $\left[s_{1}\right],\left[s_{2}\right]$. Let $C$ be the zero set of $s_{1} \wedge s_{2} \in \Gamma(L)$.

(4.3) LemMA. $C$ is an irreducible smooth hyperelliptic curve, for $s_{1}, s_{2}$ general.

Proof. As a first thing we prove that the subspace $\Lambda^{2} \Gamma(E)$ of $\Gamma(L)$ spans $L$ at every point of $X$. By contradiction, assume that there is a point $x \in X$ such that $\left(s_{1} \wedge s_{2}\right)(x)=0$ for every $s_{1}, s_{2} \in \Gamma(E)$. Then it would be $s_{1}(x)=t s_{2}(x)$ for some complex number $t$. This would mean that $\Gamma(E)$ does not span the whole fibre $E_{x}$, but just a 1-dimensional subspace of it, contradicting the spannedness of $E$. It 
follows that the sublinear system of $|L|$ corresponding to $\Lambda^{2} \Gamma(E)$ is base point free and then, in view of Bertini's theorem, $C$ is a smooth divisor, for $s_{1}, s_{2}$ general. It follows that $C$ is also irreducible. Actually, let $C=D_{1}+D_{2}$, with $D_{1}, D_{2}$ effective divisors. Since $L$ is ample, $C$ is 1-connected, hence $D_{1} \cdot D_{2}>0$, contradicting the smoothness. Finally note that $C$ consists of those points of $X$ at which the sections $a s_{1} \wedge b s_{2}$ vanish, $\left((a: b) \in \mathbf{P}^{1}\right)$. Inside $X \times|E|$ consider the incidence correspondence

$$
Z=\{(x,[s]) \in X \times \ell \mid s(x)=0\} \subset X \times|E|
$$

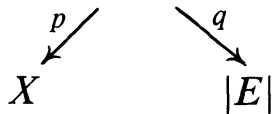

where $[s]$ is the class of $s$ and $p, q$ are the projection morphisms. Note that $p$ maps $Z$ birationally onto $C$, while $q: Z \rightarrow \ell$ is 2 to 1 . This shows that $C$ is hyperelliptic.

(4.4) Proposition. Let $(X, E)$ be as in (4.0) and $g \geq 2$. Then

(4.4.1) $\operatorname{dim} \mathscr{H} \geq h^{0}(E)-2$;

(4.4.2) $h^{0}(L) \geq h^{0}(E)$;

(4.4.3) $(X, L)$ satisfies (1.0).

Proof. Let $s_{0}, \ldots, s_{n}$ be general sections of $E$ constituting a basis of $\Gamma(E)$. In view of (4.3) the hyperelliptic locus $\mathscr{H}$ of $|L|$ contains the linear system corresponding to the subspace of $\Gamma(L)$ generated by $s_{0} \wedge s_{1}, s_{0} \wedge s_{2}, \ldots, s_{0} \wedge s_{n}$. We prove that these sections of $L$ are linearly independent. Assume, by contradiction, that $s_{0} \wedge s_{1}$, $s_{0} \wedge s_{2}, \ldots, s_{0} \wedge s_{n}$, are linearly dependent. Then there is a section $\tau=a_{1} s_{1}+\cdots+a_{n} s_{n}$ such that

$$
s_{0} \wedge \tau=0 \text {. }
$$

This means that $s_{0}=f \tau$, for some meromorphic function $f$ on $X$. But since $s_{0}$ vanishes at two points only we conclude that $f$ is a constant function and so $s_{0}$ is a linear combination of $s_{1}, \ldots, s_{n}$, a contradiction. Therefore

$$
\operatorname{dim} \mathscr{H} \geq n-1 \text {. }
$$

This proves (4.4.1). In particular $h^{0}(L) \geq h^{0}(E)-1$ and if equality holds it follows that $s_{0} \wedge s_{1}, s_{0} \wedge s_{2}, \ldots, s_{0} \wedge s_{n}$ is a basis of $\Gamma(L)$. In this case every element of $\Gamma(L)$ has the form $s_{0} \wedge \tau$ and then vanishes at the zero set of $s_{0}$. But this would mean that $L$ is not spanned, 
contradiction. This proves (4.4.2). The last assertion follows from (4.4.1) and the fact that $h^{0}(L) \geq 4$. Actually since $E$ is ample and spanned the map associated with the tautological bundle $\xi_{E}$ on $\mathbf{P}(E)$ is a finite morphism; hence $h^{0}(E)=h^{0}\left(\xi_{E}\right) \geq \operatorname{dim}(\mathbf{P}(E))+1=4$.

In view of (4.4), to get our classification we need to check all pairs $(X, L)$ listed in the previous sections. By using (4.1) we see that $(X, L)$ cannot be a scroll. As to conic bundles, we have

(4.5) LemMA. Let $(X, E)$ be as in (4.0) and assume that $(X, L)$ is a conic bundle. Then $X$ is a $\mathbf{P}^{1}$-bundle and $L \sim_{n}[2 \zeta+(e+2) f]$.

Proof. $X$ has to be a $\mathbf{P}^{1}$-bundle in view of (4.1); hence $L \sim_{n}$ $[2 \zeta+b f]$. We want to determine $b$. Since $E \otimes[\zeta]^{-1}$ restricts trivially to the fibres of $X$, we have $E \otimes[\zeta]^{-1}=p^{*} G$, where $p: X \rightarrow B$ is the ruling projection and $G=p_{*}\left(E \otimes[\zeta]^{-1}\right)$ is a rank-2 vector bundle on $B$. From $E=[\zeta] \otimes p^{*} G$ we get $L=\left[2 \zeta+c_{1}(G) f\right]$, so that $b=c_{1}(G)$. Finally

$$
2=c_{2}(E)=e_{2}\left([\zeta] \otimes p^{*} G\right)=c_{1}(G)+\zeta \cdot \zeta=b-e .
$$

It follows from (4.5) that conic bundles as in $\S 2$ cannot occur here. Moreover, due to (4.4.2), the remaining pairs coming from $\S 2$ (not fitting into (1.3)) cannot occur here as well, since $h^{0}(L)=3$ for all of them.

(4.6) Proposition. Let $(X, E)$ be as in (4.0) with $g \geq 2$ and assume that $(X, L)$ is not a conic bundle. Then there is a morphism $\pi: X \rightarrow \mathbf{P}^{2}$ of degree 2 and $E=\left(\pi^{*} \mathscr{O}_{\mathbf{P}^{2}}(1)\right)^{\oplus 2}$.

Proof. As we said $(X, L)$ is not as in $\S 2$. Moreover $(X, L)$ cannot admit nontrivial reductions by (4.1). Then there is a morphism $\pi: X \rightarrow Y$ of degree 2 and $L=\pi^{*} M$, where $(Y, M)$ is as in (1.3). Consider the morphism $\mu: X \rightarrow G$ from $X$ to the grassmannian of the codimension 2 linear subspaces of $\Gamma(E)$ sending $x$ to the subspace $\{s \in \Gamma(E) \mid s(x)=0\}$. By [GH, p. 731], $\mu$ factors through the adjunction mapping of $L$; hence, by (1.3), the vanishing of the sections of $E$ is invariant under the involution defined by $\pi$, we conclude that $\mu$ factors through $\pi$. Since $E$ is the pull back via $\mu$ of the universal rank-2 quotient bundle on $G$, it follows that there exists a rank-2 vector bundle $F$ on $Y$ such that $E=\pi^{*} F$. Moreover $F$ is ample and spanned since $E$ is so and $\pi$ is finite. Also $c_{2}(F)=1$ by the functoriality of the Chern classes. Thus $(Y, F)=\left(\mathbf{P}^{2}, \mathscr{O}_{\mathbf{P}^{2}}(1) \oplus \mathscr{O}_{\mathbf{P}^{2}}(1)\right)$, by [LS, Cor. (1.2)] and this concludes the proof. 
To complete the analysis we only need to look at conic bundles $(X, L)$ where $X$ is a $\mathbf{P}^{1}$-bundle over a smooth curve of genus $q$. Assume for a moment that $X$ is rational; then (4.5) and the ampleness condition for $L, e+2>2 e$, imply $e \leq 1$, so that $(X, L)$ can only be one of the following pairs: $\left(\mathbf{F}_{0},[2 \zeta+2 f]\right),\left(\mathbf{F}_{1},[2 \zeta+3 f]\right)$. In the former case $g=1$, while in the latter one $\zeta$ would be a line relative to $L$, contradicting (4.1). This shows that $q \geq 1$ and then by (3.2) and (3.3) we know that $g=2 q+1, d=8, e \leq 0$ and $L \sim_{n}[2 \zeta+(e+2) f]$. In fact one can prove that $\operatorname{deg} E_{\mid \zeta} \geq 3$, since $E$ is spanned. Therefore $3 \leq c_{1}\left(E_{\mid \zeta}\right)=c_{1}\left(L_{\mid \zeta}\right)=(2 \zeta+(2+e) f) \cdot \zeta=2-e$, hence $e \leq-1$. So we have proved

(4.7) THEOREM. The pairs $(X, E)$ as in (4.0) are the following:

(a) $\left(\mathbf{P}^{2}, \mathscr{O}_{\mathbf{P}^{2}}(1) \oplus \mathscr{O}_{\mathbf{P}^{2}}(2)\right)$,

(b) $\left(\mathbf{F}_{0}, \mathscr{O}_{\mathbf{F}_{0}}(1) \oplus \mathscr{O}_{\mathbf{F}_{0}}(1)\right)$,

(c) $\pi: X \rightarrow \mathbf{P}^{2}$ is a double cover and $E=\left(\pi^{*} \mathscr{O}_{\mathbf{P}^{2}}(1)\right)^{\oplus 2}$, or possibly

(d) $p: X \rightarrow B$ is a $\mathbf{P}^{1}$-bundle of invariant $e \leq-1$ over an elliptic or a hyperelliptic curve and $\operatorname{det} E \sim_{n}[2 \zeta+(e+2) f]$.

It seems difficult to decide whether case d) does really occur. In any case, if such an $E$ exists, it has the form $E=p^{*} G \otimes[\zeta]$ and is indecomposable in view of [L1].

\section{REFERENCES}

[Ba] E. Ballico, Rank-2 vector bundles on a surface with many sections and low $c_{2}$, preprint.

[F1] T. Fujita, On the structure of polarized manifolds with total deficiency one, I, J. Math. Soc. Japan, 32 (1980), 709-725.

[F2] _ On hyperelliptic polarized varieties, Tohoku Math. J., 35 (1983), 1-44.

[GH] P. Griffiths and J. Harris, Principles of Algebraic Geometry, J. Wiley \& Sons, New York, 1978.

[L1] A. Lanteri, A note on k-dimensional double solids, Rend. Sem. Mat. Brescia, 10 (1988), 1-9.

[L2] _ Variazioni sul tema classico delle sezioni iperellittiche, Rend. Sem. Mat. Fis. Milano, 57 (1987), 533-547.

[LL] A. Lanteri and E. L. Livorni, Complex surfaces polarized by an ample and spanned line bundle of genus three, Geom. Dedicata, 31 (1989), 267-289.

[LP1] A. Lanteri and M. Palleschi, About the adjunction process for polarized algebraic surfaces, J. reine und Angew. Math., 352 (1984), 15-23.

[LP2] _ Adjunction properties of polarized surfaces via Reider's method, Math. Scand., to appear.

[LS] A. Lanteri and A. J. Sommese, $A$ vector bundle characterization of $\mathbf{P}^{n}$, Abh. Math. Sem. Univ. Hamburg, 58 (1988), 89-94. 
[R] I. Reider, Vector bundles of rank-2 and linear systems on algebraic surfaces, Ann. of Math., 127 (1988), 309-316.

[Sa] F. Sakai, Ample Cartier divisors on normal surfaces, J. reine und Angew. Math., 366 (1986), 121-128.

[Se] F. Serrano, The adjunction mapping and hyperelliptic divisors on surfaces, J. reine und Angew. Math., 381 (1987), 90-109.

[So1] A. J. Sommese, Hyperplane sections of projective surfaces I, The adjunction mapping, Duke Math. J., 46 (1979), 377-401.

[So2] _ On the minimality of hyperplane sections of projective threefolds, $\mathrm{J}$. reine und Angew. Math., 329 (1981), 16-41.

[SVdV] A. J. Sommese and A. Van de Ven, On the adjunction mapping, Math. Ann., 278 (1987), 593-603.

[V] A. Verra, On Enriques surface as a fourfold cover of $\mathbf{P}^{2}$, Math. Ann., 266 (1983), 241-250.

Received June 14, 1988 and in revised form October 25, 1988. Partially supported by M.P.I. of the Italian government.

UNIVERSITÀ DEGLI STUdI DELL'AQUILA

I-67100 L'AQUILA, ITALY

AND

Dipartimento di MATEMATica "F. EnRIQues"-UUniversità

Via C. SAldini, 50

I-20133 Milano, Italy 


\section{PACIFIC JOURNAL OF MATHEMATICS EDITORS}

\author{
V. S. VARADARAJAN \\ (Managing Editor) \\ University of California \\ Los Angeles, CA 90024-1555-05 \\ Herbert Clemens \\ University of Utah \\ Salt Lake City, UT 84112 \\ Thomas ENRIGHT \\ University of California, San Diego \\ La Jolla, CA 92093
}

R. FINN

Stanford University

Stanford, CA 94305

Hermann FlaschKa

University of Arizona

Tucson, AZ 85721

VAughan F. R. Jones

University of California

Berkeley, CA 94720

Steven Kerckhoff

Stanford University

Stanford, CA 94305
RobION KIRBY

University of California

Berkeley, CA 94720

C. C. MOore

University of California

Berkeley, CA 94720

HAROLd STARK

University of California, San Diego La Jolla, CA 92093

\begin{tabular}{|c|c|c|c|c|}
\hline & ASSOCIA & E EDI & & \\
\hline R. ARENS & $\begin{array}{l}\text { E. F. BECKENBACH } \\
(1906-1982)\end{array}$ & NeumanN & $\begin{array}{c}\text { F. WoLF } \\
(1904-1989)\end{array}$ & K. Yoshida \\
\hline & SUPPORTINC & INSTI & TIONS & \\
\hline UNIVERSITY & ARIZONA & UNIVERS & OF OREGON & \\
\hline UNIVERSITY & BRITISH COLUMBIA & UNIVER & OF SOUTHEI & CALIFORNIA \\
\hline CALIFORNIA & STITUTE OF TECHNOLOGY & STANFOI & UNIVERSITY & \\
\hline UNIVERSITY & CALIFORNIA & UNIVER & OF HAWAII & \\
\hline MONTANA S & TE UNIVERSITY & UNIVER & OF TOKYO & \\
\hline UNIVERSITY & NEVADA, RENO & UNIVER & OF UTAH & \\
\hline NEW MEXIC & TATE UNIVERSITY & WA & N STATE UN & ERSITY \\
\hline OREGON ST & UNIVERSITY & & OF WASHINC & $\mathrm{ON}$ \\
\hline
\end{tabular}




\section{Pacific Journal of Mathematics}

\section{Vol. 143, No. $1 \quad$ March, 1990}

Walter Bergweiler, On the fix-points of composite functions $\ldots \ldots \ldots \ldots \ldots 1$ Aldo Biancofiore, Maria Lucia Fania and Antonio Lanteri, Polarized surfaces with hyperelliptic sections $\ldots \ldots \ldots \ldots \ldots \ldots \ldots \ldots \ldots$

Ciprian Borcea, Deforming varieties of $k$-planes of projective complete

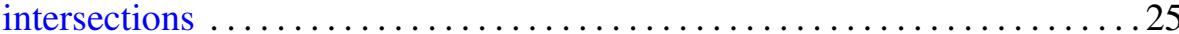

Morton Brown, Fixed points for orientation preserving homeomorphisms of

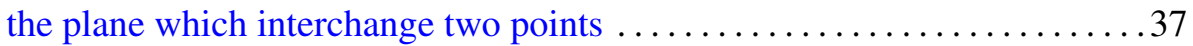

Hao Zhi Chuan, Note on the inequality of the arithmetic and geometric means

Paul Henry Edelman and Dennis E. White, Codes, transforms and the

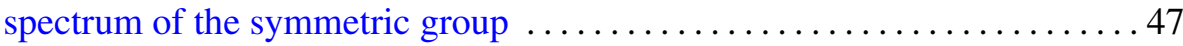

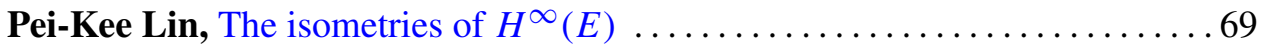

James J. Moloney, Residue class domains of the ring of convergent

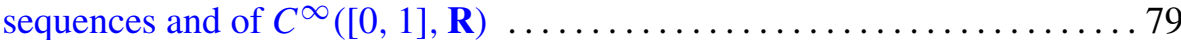

Zhong-Jin Ruan, The structure of pure completely bounded and completely

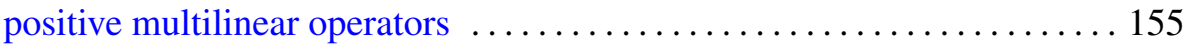

Wolfgang Ruess and William H. Summers, Weakly almost periodic semigroups of operators

Gideon Schwarz, A pretender to the title "canonical Moebius strip" . . . . . . 195

Ryszard Szwarc, Banach algebras associated with spherical representations of the free group 